\title{
Agencia femenina en los procesos migratorios internacionales: Una aproximación epistemológica
}

\author{
Women's agency in international migration processes: \\ An epistemological approach
}

\author{
MÀrius Domínguez Amorós \\ Paola Contreras Hernández \\ Universitat de Barcelona \\ mariusdominguez@ub.ed (ESPAÑA)
}

Recibido: 28.10 .2016

Aceptado: 31.03 .2017

\section{RESUMEN}

Este artículo tiene por objetivo analizar cómo el colectivo de mujeres latinoamericanas que viven en Barcelona, despliega su capacidad de agenciamiento, considerando la manera en que los procesos de inclusión y exclusión tienden a constituir determinadas sinergias que las sitúan como sujetos activos dentro del devenir migratorio. El sustento teórico viene dado por las distintas aportaciones que se han realizado en el área de los estudios migratorios, principalmente aquellos que han utilizado el género como categoría analítica y los que han incorporado la perspectiva transnacional. Asimismo, se presentan los enfoques que han teorizado sobre los procesos de exclusión e inclusión, para luego introducir el concepto de agencia en clave feminista. De este modo, y a partir de un modelo de análisis que surge de la teoría y del trabajo empírico realizado con veinticinco mujeres latinoamericanas, en la ciudad de Barcelona entre los años 2015 y 2016, se observan distintas formas en que las mujeres fortalecen su agencia y que les permite construir espacios asociativos para la sobrevivir y prosperar social, económica y personalmente.

\section{PALABRAS CLAVE}

Migraciones internacionales, Procesos inclusión/exclusión social, Género, Estrategias, Agencia. 


\begin{abstract}
This article aims to analyze how the community of Latin American women living in Barcelona display their agency ability, considering how processes of inclusion and exclusion tend to constitute certain synergies that place them as active subjects in the migration becoming. The theoretical framework is developed from the main contributions that have been done in the area of migration studies, focusing mainly on those that have used gender as an analytic category and those that have incorporated a transnational perspective. In addition, approaches that have theorized about the processes of exclusion and inclusion are presented, then the concept of key feminist agency is introduced. Thus, and from an analysis model that emerges from the theoretical and empirical work with twenty-five Latin American women in Barcelona, Spain, between 2015 and 2016, different ways are observed in which women strengthen their agency, which allows them to build associative spaces in order to survive and thrive socially, economically and personally.
\end{abstract}

\title{
KEY WORDS
}

International migration, Processes social inclusion/exclusion, Gender, Strategies, Agency.

\section{INTRODUCCIÓN}

Desde distintas teorías y enfoques metodológicos, las investigaciones científicas se han aproximado al fenómeno migratorio, generando un cúmulo de conocimiento que ha permitido comprender la complejidad del proceso. En la actualidad y bajo el contexto de la globalización, el interés académico se ha centrado en investigar los distintos factores que inciden en la migración, sus características y tendencias actuales. Es en este contexto que uno de los ejes que desde hace un tiempo se viene profundizando se relaciona con la migración femenina; feminización que para Castles y Miller (1998) marca un nuevo ciclo en el estudio de las migraciones debido al protagonismo que las mujeres están asumiendo, y que la lleva a constituirse como un desafío social, científico y epistemológico.

La literatura científica muestra que se ha avanzado considerablemente en el cuerpo teórico sobre las dinámicas femeninas en temas relacionados con las causas que originan la migración, con las consecuencias que ello implica para las familias y en las propias mujeres que lo asumen, con la dimensionalidad de lo transnacional (cadenas globales de cuidados, por ejemplo), con las tensiones que enfrentan en el país de destino, con los procesos de integración, entre otros temas. Sin embargo, consideramos que existe una categorización que tiende a homogenizar tanto sus historias como sus experiencias, lo que impide analizar 
las estrategias emprendidas para alcanzar los propósitos/objetivos que originaron dicha movilidad internacional.

Por ello, este artículo tiene por objetivo analizar el proceso de agenciamiento que despliega el colectivo de mujeres latinoamericanas en Barcelona. Con ello se busca abordar un eje poco explorado que pretende identificar cómo, dentro de los procesos de inclusión y exclusión, se van constituyendo determinadas sinergias que logran situar a las mujeres como sujetos activos dentro del devenir migratorio y que permite debilitar estereotipos que se han erigido sobre sus cuerpos e identidades. Para ello se presentan, en primer lugar, los principales ejes teóricos que han dado sustento a los estudios migratorios femeninos, lo cual nos permitirá visibilizar - de manera general- los aspectos que han concentrado mayor interés en las investigaciones científicas. En segundo lugar, se abordan desde el enfoque transnacional, aspectos relacionados con las motivaciones, experiencias, trayectorias y estrategias, con la finalidad de mostrar la heterogeneidad que presentan. En tercer lugar, se reflexiona sobre los enfoques analíticos que han buscado definir los procesos de exclusión e inclusión; para luego situar el concepto de agencia en clave feminista. Por último, se presenta un modelo de análisis (que surge del análisis teórico y de los hallazgos preliminares del trabajo empírico) que visualiza cómo al tensionar los procesos de exclusión e inclusión a los que se ven enfrentadas las mujeres migrantes, emerge un espacio donde es posible localizar la capacidad de agenciamiento, y que se expresa, por ejemplo, en la construcción de espacios asociativos para la supervivencia social, económica y personal.

\section{FEMINIZACIÓN DE LAS MIGRACIONES: CONTRIBUCIONES Y DEBATES}

En los actuales estudios sobre migración femenina hay autoras como Gregorio Gil (1998) que establecen una diferenciación entre lo que es la feminización y generización de las migraciones, indicando que esta última

Surge de la adopción de una perspectiva feminista en el estudio de las migraciones, al contemplar la influencia de los sistemas de género como principios organizadores de ésta, [en cambio feminización] pone el énfasis en el estudio de un sector de la sociedad -las mujeres- y da cuenta de un fenómeno creciente en importancia en términos de número, cuyas raíces pueden llevar o no a un análisis feminista (Gregorio Gil 1998: 263).

Por su parte, Tapia (2011) señala que las migraciones internacionales pueden ser vistas como un proceso generizado por el potencial heurístico que tiene el género en cuanto a su carácter categorial y analítico. Esta distinción muestra cómo dentro de los estudios sociales se van marcando diferencias a la hora de abordar un tema tan relevante como son las migraciones; pero más allá de eso, quienes han profundizado en este tema constatan el protagonismo que las mujeres están asumiendo, redefiniéndolas como un actor central (Magliano, 
Romano 2009). Este protagonismo manifiesta un cambio respecto al patrón de movilidad: las mujeres han dejado de ser parte de un proceso asociativo (reagrupación familiar), para pasar a uno de carácter autónomo que responde a estrategias familiares y personales. Con todo, dicho brevemente, la feminización de las migraciones se circunscribe en un escenario global de transformaciones sistémicas; es por ello que, al considerar el género como un elemento central, es necesario analizarlo en intersección con otros factores que actúan e inciden en este proceso. En este sentido, enfatizar el prisma económico puede llevar a sesgos analíticos; de ahí la importancia de conjugar diversos factores que entran en esta dinámica, por ejemplo, la violencia patriarcal, la desigualdad de género, el desarrollo de las comunicaciones y nuevas tecnologías, las redes migratorias, etc. (Gil Araujo 2005).

En este contexto, Hondagneu-Sotelo (2011) plantea seis enfoques vinculados a las investigaciones sobre género y migración que en la actualidad prevalecen. A nuestro interés, tres de ellos son relevantes ${ }^{1}$. El primero busca hacer del género una parte constitutiva de las investigaciones sobre migración, comprendiendo su alcance en la promoción de una lectura en cuanto a cómo la diferencia sexual actúa e incide en los procesos personales y sociales. Desde esta premisa, el género como categoría de análisis ha comenzado a ser incorporado en las distintas áreas del proceso migratorio (Hondagneu-Sotelo 2007): el mercado laboral (Catarino, Oso 2000); las relaciones familiares; la maternidad y familia transnacional (Parella, Cavalcanti 2010; Sinatti 2008); las tensiones patriarcales en cuanto a los cambios o modificaciones en los roles de género (Martín 2012); las políticas y control migratorio (Calavita, 2006); la violencia machista (Montañez, Moyano 2006); y la feminización de las migraciones (Tapia 2011), entre otros.

El segundo enfoque se centra en el trabajo doméstico y de cuidados, el cual es conectado con las aportaciones realizadas desde el paradigma de la interseccionalidad, ya que evidencia cómo los distintos modelos de opresión (sexismo, racismo, clasismo, etc.), van configurando desigualdades múltiples. A su vez, debilita la imagen esencialista de lo que era ser hombre o mujer, puesto que "fueron reemplazados con la idea de que hay multiplicidad de feminidades y masculinidades, y que están interconectadas, relacionadas y entrelazadas con las desigualdades de clase, raza, etnia, nación y sexualidades" (Hondagneu-Sotelo 2011: 221-222). En esta línea, destacan investigaciones en torno a las cadenas globales de cuidados (Carrasco 2013; Herrera 2012) y el trabajo doméstico (Escrivá, 2000; Hondagneu-Sotelo, Avila 1997), etc.

El tercer enfoque de interés en nuestro marco analítico aborda la trata de personas para fines sexuales y laborales, profundizando en el trabajo sexual que

${ }^{1}$ Los tres enfoques restantes son los que se orientan, primero, al estudio de la sexualidad y las identidades de género, que en principio se centró en las identidades queers y gays, pero hoy abarca la hetero-normatividad obligatoria y como ello actúa como mecanismo de exclusión o inclusión (Gregorio Gil 2009). Segundo, aquellos relacionados con las fronteras e hibridez (Anzaldúa 1987). Por último, están las perspectivas que sitúan la infancia y que suelen estar vinculados con dinámicas transnacionales. 
de manera libre ejercen las mujeres migrantes y que es encasillado dentro de la economía informal (Acién 2009). Este tema no deja de ser controvertido por la connotación moral que tiene, porque además, imposibilita ver cómo las mujeres inmigrantes enfrentan la explotación en otros nichos laborales.

Estos enfoques permiten visibilizar una imagen general sobre la manera en que se ha abordado el género en los estudios migratorios, que tal y como señala Gregorio Gil (1999) en un principio fue excluido principalmente por el carácter androcéntrico de los instrumentos que recogen información sobre el tema, pero también por una connotación ideológica que no reconoce la presencia de las mujeres más allá del espacio doméstico-privado. Esta situación comenzó a cambiar desde mediados de la década de los ochenta al incorporar el género como categoría analítica, donde "la variable género, más allá de la variable sexo, se convierte en un factor de análisis en sí mismo" (Aubarell 2000: 392). Este factor permite incorporar y recuperar en las investigaciones las experiencias femeninas, visibilizándolas y dándoles valor social y político.

Por tanto y desde estas contribuciones, el género es un elemento constitutivo y estructurante de la migración que necesariamente debe ser vinculado con otras dimensiones, factores, prácticas e identidades; una intersección que permite analizar cuáles son y cómo actúan las estructuras de opresión sobre las mujeres. Es justamente en este punto que aquellas investigaciones que recogen e intersectan el género, la raza, la clase, la sexualidad y la procedencia han tomado relevancia epistemológica. Cabe añadir que sin lugar a dudas los estudios sobre migración y género han alcanzado un desarrollo teórico, metodológico y analítico que permite avanzar en ámbitos muy significativos tanto en la vida de las propias mujeres que emigran, como en la de sus familias, en las transformaciones de los mercados laborales, en las relaciones de género, etc.

\section{MOTIVACIONES, PROYECTOS, ESTRATEGIAS: UN ACERCAMIENTO DESDE EL ANÁLISIS TRANSNACIONAL}

En la actualidad, el transnacionalismo se ha constituido tanto en un marco analítico que ha propuesto nuevos conceptos, categorías y enfoques, como en un enfoque polisémico que ha llevado a plantear algunos interrogantes epistemológicos (Briggs, McCormick, Way 2008). Sin embargo, una de las características que aúna las distintas aproximaciones teóricas de lo transnacional es su bifocalidad, la cual "implica de alguna manera al que se va como al que se queda, transformando la percepción sociocultural de ambos grupos, pues con ésta surgen nuevos estilos y prácticas cotidianas más "hibridas" y se modifican los significados y valores" (Bobes 2012:10). Así, las comunidades transnacionales sitúan las migraciones no sólo como un cambio de residencia, sino como un vivir transnacional que se forja a partir de relaciones transfronterizas y que "emerge, intencional e inesperadamente, de la presión de los migrantes por mantener y reproducir su ambiente socio-cultural de origen a distancia" (Guarnizo 2004:15). Dentro de las dinámicas globalizadoras, los 
múltiples posicionamientos de ser un sujeto transnacional y las consecuencias que ello tiene en ambos lados de las fronteras, proyectan un nuevo espacio social -entendido como "una construcción social analítica que no tiene que ver con lo espacial" (Jiménez 2010: 19)-; una pertenencia dentro de un campo de relaciones que implica el desarrollo de prácticas y relaciones entre grupos específicos que se vinculan por afinidad e identidad, lo cual otorga una forma transnacional de ser en el momento en que se reconocen dichas prácticas (Levitt y Glick Schiller 2004; Portes 1999; Sinatti 2008).

En un sentido más amplio, para Jiménez (2010) una de las cuestiones espinosas a resolver es cómo se puede pensar y entender al sujeto femenino bajo estas consideraciones. Para Segura y Zavella (2007), las mujeres tienden a adaptarse a las transformaciones que conlleva la experiencia migratoria, lo que implica una resignificación de sus identidades más allá de las representaciones sociales que se edifican sobre sus cuerpos; recomponiendo con ello su voz frente a sus experiencias. Es así que descifrar las dinámicas de los mecanismos que tienden a diferenciar a la población inmigrante y que perpetúa asimetrías en la estructura social, política y económica ha sido uno de los desafíos de las ciencias sociales en los últimos años. De ahí la crítica a la construcción homogénea que se ha hecho de la mujer migrante, un arquetipo que se define desde la fragilidad, vulnerabilidad e indefensión y que funda estereotipos y estigmatizaciones que invisibilizan su heterogeneidad y niegan su capacidad agencial. Es en este contexto que las aproximaciones metodológicas y epistemológicas obligan a pensar nuevas lecturas hacia los cuerpos migrados, replanteándose conceptos y categorías con las que se han investigado las migraciones femeninas. Dentro de estos pueden destacarse las trayectorias de vida, ya que confieren una perspectiva diacrónica de tales procesos, que al vincularla con las motivaciones, las estrategias, la agencia y las resistencias, permiten ir configurando un mapa más amplio y diverso, no sólo del por qué las mujeres emigran, sino también cómo enfrentan los desafíos al instalarse en el país de llegada/destino y cuáles son las consecuencias o efectos que ello tiene en sus vidas e identidades. Para Bourdieu (1977) las trayectorias son circunstancias que desarrollan los agentes en espacios diversos y en constante transformación; la reconstrucción de pasajes, momentos o procesos en la vida de los sujetos investigados permiten comprender la importancia que ciertos acontecimientos han tenido en sus vidas y que -de alguna manera- han determinado el rumbo de sus biografías.

En cuanto a las trayectorias migratorias, para Micolta (2005) éstas varían según el tiempo (estacional, temporal, indefinidas); el modo de vida, es decir, de acuerdo a las demandas personales (búsqueda de estatus, por actividad profesional, desarrollo académico); la edad (infantil, adultos o ancianos); y por el grado de libertad (voluntarias, forzadas, refugiados, deportados). Si bien pueden existir características diversas entre distintos colectivos de inmigrantes, las motivaciones y necesidades confluyen y se yuxtaponen por causas económicas, políticas, por necesidad de promoción personal, por violencias machistas, sociales y/o políticas, por reagrupación familiar, etc. En esta línea, tal y como señala Gil Araujo (2005), el incremento de las 
mujeres en los circuitos alternativos de supervivencia ha sido consecuencia de la aplicación de políticas neoliberales ${ }^{2}$. Esta incorporación ha supuesto un cuestionamiento al modelo hegemónico masculino que sitúa al hombre como principal sostenedor económico del núcleo familiar (Godoy 2007; Parella 2012; Tapia 2011). No obstante, las motivaciones económicas son insuficientes para explicar en su totalidad la migración, siendo necesario incorporar "factores sociales y culturales que no necesariamente responden a una lógica económica racional. "Imaginarios", "redes sociales", "cadenas horizontales y verticales" y "estrategias familiares" son las herramientas conceptuales que se despliegan para ofrecer una interpretación social y cultural antes que económica de la migración" (Herrera 2013: 89-90). Además, centrar el análisis sólo en la dimensión económica invisibiliza otras esferas que actúan en el proceso de feminización de las migraciones, por ejemplo, las relaciones de género, "las que en el caso de las migraciones latinoamericanas, a menudo constituyen "una forma de exclusión social en el contexto de origen que motiva a las mujeres a emigrar" (Godoy 2007: 42).

Dicho esto, observamos cómo las motivaciones permiten ir definiendo determinadas estrategias migratorias; estrategias que se diferencian según el género y que para las mujeres están determinadas por la responsabilidad de subsistencia económica del núcleo familiar y por la configuración de un mercado laboral que las segrega al nicho reproductivo. Por su parte, Martín (2012) y Oso (2003) señalan que a la hora de estudiar las estrategias migratorias es necesario partir de aquella que busca soslayar los controles fronterizos, que -en muchos casos- se ciñe a la figura de turista en el momento de llegada al país de destino, frecuentemente utilizada por mujeres latinoamericanas, encubriendo su propósito de quedarse. Luego, una vez instaladas, las estrategias se orientan a resolver los problemas de subsistencia, los aspectos organizativos de la vida cotidiana, comenzar a pagar las deudas del viaje o para iniciar el envío de remesas a sus familias. Por ello, establecer contactos con redes sociales y/o familiares es una estrategia fundamental para encontrar trabajo. Sin embargo, la premura laboral puede tener un impacto en el descenso socio-laboral, principalmente porque el servicio doméstico -independiente del nivel formativo/profesional de la migrante- se presenta como la opción más concreta de trabajo, lo cual refleja el nivel de segregación y devaluación de esta labor (Dumitru 2014). Las mujeres migrantes suelen justificar el descenso social que les significa su nueva actividad laboral mediante una lectura pragmática de los beneficios que ello les reporta.

Por último, cabe añadir que en esta etapa las tensiones emocionales se hacen más fuertes, originando un duelo y/o limbo migratorio, el cual influirá en el primer momento del proceso de instalación, el que, a su vez, va gestando dimensiones de lo transnacional por la conformación de posiciones multisituadas/localizadas (el aquí y el allá). De igual modo, en este periodo es cuando se observa un proceso de "integración" a la nueva cultura; aunque también es

${ }^{2}$ En América Latina se comienzan a desarrollar con la aplicación de los planes de ajustes estructurales (PAE). 
posible que se origine una "acomodación", es decir, una aceptación mínima a la cultura del país de llegada/destino al no existir un deseo profundo de incorporarse en ella, la cual no llega a ser conflictiva en su totalidad (Micolta 2005). Es en este punto donde surgen interrogantes en torno a cómo el contexto social influye en el proceso de integración, considerando las dificultades que muchas veces deben enfrentar a propósito de las tensiones que se generan en los procesos de inclusión y exclusión.

Es importante destacar que estos momentos, periodos o etapas, si bien permiten tener una perspectiva longitudinal, se van asociando e intersectando entre sí, pues no son estáticos, sino dinámicos, debido a que al mezclarse lo emocional, racional y circunstancial, se originan nuevas situaciones y escenarios. Por tanto, si consideramos estas etapas, resultan ser coherentes los conceptos mencionados para aproximarnos no sólo al momento previo de la migración (trayectorias y motivaciones), sino también a la manera en que han enfrentado su nueva realidad, donde las estrategias se colocan como elemento central, pues permiten recorrer las distintas formas en que se fortalece su agencia y sus resistencias identitarias.

A continuación se presentan los principales ejes que definen los procesos de exclusión e inclusión social, pues para analizar sus estrategias y capacidad de agenciamiento, es importante considerar los elementos que lo facilitan u obstaculizan.

\section{EXPLORANDO EN EL BINOMIO EXCLUSIÓN/INCLUSIÓN DE LA EXPERIENCIA MIGRATORIA}

El planteamiento de la visión transnacional al análisis de los procesos migratorios implica plantear la construcción política de la ciudadanía, y la manera en que se establecen procesos de diferenciación entre las personas migrantes y los nacionales. Estos procesos proyectan una frontera simbólicajurídica dentro de un mismo territorio; lo cual determina que quien está dentro tiene derechos y libertades, y quien está fuera sólo tiene obligaciones (Carrera, 2006). Y es desde esta perspectiva que, al investigar sobre las migraciones, se plantee como relevante incidir y problematizar los procesos de inclusión y excusión social.

En este contexto, la (re)definición de la exclusión social va más allá de la pobreza y desigualdades económicas, ya que su definición apunta a un proceso de pérdida de integración política y social, una carencia de bienestar que se relaciona no sólo con el desempleo y la pobreza económica, sino también con la privación de vínculos sociales que conlleva una disgregación, provocando una ruptura progresiva de la relación entre el individuo y la sociedad (VII Informe Foessa 2014; Laparra et al., 2007; Silver 1995). Sin embargo, para Fleury (1998), la falta de consenso teórico ha derivado en definiciones tautológicas que sitúan esta realidad desde la carencia económica, sanitaria, educativa, de ciertos bienes y servicios, la falta de oportunidades, etc. Si bien estas dimensiones son 
parte de la exclusión, la autora critica que el énfasis está puesto en el carácter individual,

De alguna manera se trata de buscar un conjunto de características y/o atributos que limitarían la posibilidad de inserción de los individuos en el mercado, ya sea como productores o como consumidores. En este sentido, esas definiciones circunscriben el fundamento de la exclusión a su componente económico (Fleury 1998: 8-7).

Por tanto, desde el carácter multidimensional y estructural, se considera como un proceso dinámico de ruptura de los lazos sociales a nivel individual y colectivo, un quiebre en las relaciones que generan pertenencia y fortalecen la cohesión social, pues impide la participación plena en la estructura social, limitando las aspiraciones personales de quienes se encuentren en esta situación al verse aquejados por la falta de acceso a los recursos, a la información, etc. Por ello, cuando hablamos de personas o colectivos que están al margen, es decir, que no gozan de una ciudadanía social plena, se infiere que otras/os si lo hacen; lo que desde un enfoque cartesiano define una realidad dual en la que existe un sector integrado y otro excluido (Laparra et al., 2007; Subirats, Gomà, Brugué 2005).

Dentro de esta discusión, Laparra et al. (2007), señalan que existen tres enfoques posibles para explicar y aproximarse a la exclusión; el primero gira en torno a quienes se auto-excluyen por no querer seguir las pautas socioculturales impuestas; el segundo es una respuesta a los cambios socioeconómicos que surgen en el momento que se comienza a transitar de una sociedad industrial a una postindustrial y que incrementó la pobreza y marginación social; y el tercero responde a la discriminación a ciertos colectivos sociales estigmatizados, negándoles o limitando las oportunidades.

Sin embargo, cuando la exclusión es concebida como la pertenencia a un grupo específico, con lógicas, valores y conductas claramente diferenciadas de las de la sociedad mayoritaria es muy fácil que acabe reforzando esta estigmatización que en nada ayuda a vencer la exclusión (Laparra et, al. 2007: 25).

En este último caso, podemos pensar y situar a las personas migradas que se instalan en el país de destino y que desde esta lógica son excluidas por no representar los valores de la sociedad a la cual llegan, lo que puede fomentar el ascenso del racismo y la criminalización del/hacia el otro/a (Laparra 2007).

En cuanto a los procesos de inclusión social, podríamos señalar que desde una perspectiva dialéctica, sería la antítesis de exclusión. Pues bien, para autores como Subirats et al. (2010), la inclusión social se percibe mientras exista participación activa en tres áreas importantes: primero, en la economía por medio de la producción de valor social (dentro o fuera del mercado); segundo, en la política a través del ejercicio de la ciudadanía; tercero, en la esfera social y familiar mediante la pertenencia comunitaria. En este sentido, una de las cuestiones centrales en los debates sobre inclusión hace referencia a la búsqueda 
de mecanismos institucionales que permita que las personas y/o colectivos que se encuentran excluidos puedan acceder a las oportunidades y beneficios sociales (González y Güell 2012). Esta perspectiva ha sido criticada por su lógica tecnócrata, en cuanto a que sería la racionalidad y cálculo del beneficio el catalizador para que éstos actúen. De ahí que las políticas de inclusión social deberían proponer (más allá de las oportunidades), dispositivos culturales que faciliten la apropiación y pertenencia del entorno social.

Estos argumentos plantean en sus análisis dos conceptos que generan cierta controversia debido a su carácter polisémico, pero que pueden ser un ancla para (re)pensar la inclusión desde un andamiaje más amplio: la cohesión y el capital social. Si partimos del concepto de cohesión social, vemos que es ubicada como una herramienta que permite que los menos favorecidos (pobres y excluidos) se integren a la sociedad de mercado. No obstante, para otros la cohesión social se vincula con la integración, pues intenta mantener los vínculos y pertenencia social que respete la diferencia. Por otra parte, la cohesión social se caracteriza por constituirse en relación a las interacciones sociales y porque desde estas relaciones se desprenden determinados beneficios o recursos que pueden ser de tipo económico, educativo, cultural, social, etc. El capital social facilita, una vez constituida una red de contactos, definir espacios de integración, confianzas y reciprocidad, generando beneficios personales y/o colectivos debido a la seguridad que ello otorga (Alcaide 2013; Coleman 1990). Para Bourdieu (1985), las redes sociales que se establecen entre individuos y las ganancias que de ello obtienen es lo que se define como capital social. Sin embargo, para autores como Portes (1999), esta definición -que ha sido instrumentalizada en cuanto a los beneficios que los individuos obtendrían al participar en determinado grupo-, se reduce y limita a una connotación meramente cuantitativa.

Si contextualizamos la inclusión social desde la realidad migratoria, observamos distintos modelos de integración que han permitido desarrollar políticas pensadas en la comunidad migrante. Uno de estos es el multiculturalista, cuya base se orienta al respeto y protección de la diversidad cultural. Otro es el asimilacionista, el cual busca la adaptación a los valores del país de llegada y que reclama al inmigrante que renuncie a su identidad nacional, lingüística y/o cultural. Por último, se encuentra el modelo de exclusión o separación, que para Carrera (2006), se consagra por un rígido marco jurídico hacia la inmigración que busca salvaguardar la cohesión e identidad cultural.

Desde estos modelos, pero principalmente a partir del último, surge la discusión en torno a la inclusión subordinada (Brandariz 2009; De Giorgi 2010), concepto que pretende mostrar que si bien subyace una racionalidad punitiva hacia la migración en términos concretos, la forma en que se gestiona está orientada

A un esquema laboral en el que al migrante se le reservan ocupaciones caracterizadas tanto por su naturaleza imprescindible cuanto por elevadas tasas de precariedad y de explotación. En suma, a los migrantes se aplica la vertiente más severa del nuevo régimen de workfare, en el que se afirman 
segmentaciones del mercado de trabajo en clave étnica, en la medida en que las actividades de valor añadido alto o medio tiende a ser reservado para la fuerza de trabajo autóctona (Brandariz 2009: 294).

Por tanto, lo que se evidencia es que existe una hibridación en cuanto a exclusión e inclusión subordinada.

\section{AGENCIA: UNA APROXIMACIÓN TEÓRICA EN CLAVE FEMINISTA}

A propósito de las estrategias y de las posibilidades que éstas otorgan a las mujeres para desarrollar o enfrentar el nuevo contexto social, emerge un tema no menor y de gran importancia: la capacidad de agenciamiento. Autoras como Alkire (2005) plantean la discusión en torno a las diferencias entre conceptos que tienden a ser utilizados como sinónimos: agencia y empoderamiento. La agencia hace referencia al valor intrínseco que se despliega en el momento de actuar frente al planteamiento de uno o múltiples objetivos; incluye un poder efectivo para alcanzar las metas establecidas; se relaciona con la capacidad y disposición para actuar en función de los propósitos individuales y colectivos; valora las metas que se han fijado y los compromisos establecidos; implica responsabilidad en la toma y ejecución de las decisiones (Alkire 2008). Por su parte, el empoderamiento actuaría como un subconjunto de la agencia y cuyo valor instrumental se refiere a la implementación de distintos métodos para lograr la consecución de tales propósitos (Casado, 1999).

Desde un enfoque feminista, la agencia no puede ser analizada sin considerar el contexto en el cual se desarrolla, ya que permite entender cómo las mujeres van asumiendo la responsabilidad y conocimiento en el propio espacio de enunciación, es decir, recuperar la voz y ser consciente de su accionar, asumiendo los efectos/consecuencias que ello puede acarrear en sus vidas como en las personas cercanas (Casado, 1999). En alusión a ello, Spivak (1998) profundiza en la noción de subalternidad, señalando que la violencia epistémica -que surge con la historia colonial- construye, define y determina a un "otro" como un ser "anulado" en su subjetividad; de ahí que si en la producción colonial el individuo subalterno no tiene historia ni voz, cuando el subalterno es mujer la situación se torna más compleja. Por ello la autora se pregunta ¿Puede realmente hablar el individuo subalterno? Su respuesta la sitúa en necesidad del desaprendizaje de los patrones en los que se ha inscrito a la mujer subalterna y que ha invisibilizado su historia, ritos y costumbres; sólo desde la desfetichización y reconocimiento de su voz, consciencia e historia la subalterna podrá ejercer su derecho a ser reconocida y recuperar su lugar en la enunciación.

Para pensar la agencia es necesario considerar las estrategias de resistencias que se despliegan a nivel estructural, pues éstas enfrentan -en cierta medida- el poder y la manera en que determina los modos de ser en la realidad. Cuando se produce resistencia, subversión o resignificación contra la represión y 
subordinación se transforma el propio habitus. Sin embargo, para Mahmood (2008) la resistencia es una forma de acción que no implica necesariamente un fortalecimiento de la agencia, ya que si bien ésta es una respuesta a las relaciones de poder, ello no significa que se direccione de manera única a subvertir las normas, sino que también es posible performarlas y habitarlas de otras maneras. Pero más allá de la discrepancia que pueda existir entre agencia y resistencia, el movimiento feminista ha desarrollado una agenda donde ha primado la búsqueda de bienestar y el fortalecimiento de la capacidad de agenciamiento a través de la facultad de decidir y resolver de manera autónoma una acción, ya que "cuando las personas no son capaces de ejercer la agencia, pueden ser alienadas de su comportamiento, obligadas a mantener una situación sumisa, deseosa de complacer, o simplemente pasiva" (Alkire 2008: 3-4). Por tanto, la agencia entendida como un proceso de deliberación consciente e intencional, que busca conseguir uno o más objetivos, sitúa a las mujeres como sujetos activos de cambio y transformación.

Desde otro ángulo, el sujeto político del feminismo (occidental) comenzó a ser cuestionado por su carácter homogéneo y universalista, instalando la urgencia de pensar la multiplicidad de sujetos que representan las mujeres, pues ello permitirá -en teoría- transitar desde el sujeto político la -Mujer- a la agencia de las (otras) mujeres (Trujillo Barbadillo 2009). Es en este contexto que Casado (1999), al preguntarse cómo el feminismo ha construido al sujeto en sus discursos, busca delinear el dinamismo y polifonía que éste presenta, más que desarrollar una genealogía o construcción taxonómica. Para delinear una respuesta, la autora recorre las propuestas feministas comenzando con el de la igualdad y su idea sobre el sujeto femenino, indicando que éste se encuentra presente dentro de una práctica reivindicativa que se define "dentro de una lógica que podríamos denominar de Otredad, o sea, de afirmación de un colectivo por oposición a su contrario" (Casado, 1999:73). En cambio en la etapa de la diferencia, si bien el constructo no era uniforme, se estableció que entre hombres y mujeres existe una posición social y una subjetividad diferentes. El viraje que enfrenta la construcción de la praxis feminista lleva a la autora a plantear que el sujeto del feminismo de la igualdad y diferencia era un sujeto estático, ficticio y objetivado y que está sometido a una identidad inmutable.

En este sentido, y desde una lectura más allá de la igualdad o diferencia, si bien el concepto mujeres es utilizado, se prefiere hablar de agencia en cuanto a que representa una forma de actuar, de ser y que permite adaptarse a la realidad que se busca describir. Los cuestionamientos a este sujeto monolítico comenzaron a

Ser cuestionado por las voces que "desde los márgenes" del feminismo, hablan de la(s) realidad(es) de la diversidad de las mujeres (en plural). O, dicho con otras palabras, de la agencia (o agencias), o capacidad de actuación, de intervención en lo público-político, de unos sujetos autónomos (Trujillo Barbadillo, 2009:162). 
Son los feminismos periféricos/feminismos otros (Medina Martín, 2013), que objetan de manera directa la construcción inmutable y esencialista del concepto mujer, pues no permiten observar las diferencias que existen entre las mujeres; diferencias que se producen por el cruce y/o presencia de dimensiones como la clase, la raza, la opción sexual, la edad, y de aquellas que son migrantes y las que no, y que van definiendo una matriz de opresión y desigualdad. Esto subyace a lo que algunas feministas han definido como política de localización, la cual busca debilitar las construcciones ontológicas del sujeto mujer para así reconocer y asumir el propio lugar en la enunciación. La política de localización se funda en reconocer diferencias y asimetrías entre las mujeres a través de sus cuerpos, identidades y subjetividades; arremetiendo como un campo epistemológico necesario a explorar que reivindica una resignificación del género en base a su posicionalidad (Casado 2003). Es justamente desde esta posición que la mediación entre mujeres será fructífera debido a que las diferencias (y no la diversidad) serán la base para establecer un locus feminista amplio e incluso divergente, pero que apuesta por aceptar experiencias situadas desde la propia existencia (Lecinana 2004). Ello daría lugar a la construcción de un acervo feminista que, asume la historia y las vivencias encarnadas y corporizadas desde una política y epistemología localizada y posicionada desde la parcialidad. Desde estos postulados, hablar de subjetividad

Es hablar de la condición de los sujetos, de su índole, de su peculiaridad, de aquello que los delimita y distingue del mundo de los objetos. El concepto de subjetividad alude a la posibilidad de algunos seres vivos de tomar conciencia acerca de su condición, esto es, de volver su sensibilidad y potencial reflexivo sobre ellos mismos, percatándose de su realidad distinta de la de otros seres animados e inanimados; en este sentido, hablar de "sujetos" es, por definición, hablar de seres humanos (Ruiz 1998: 143).

Por tanto, la subjetividad femenina implica pensar cómo se constituyen los significados y se resignifica la realidad vivida y hasta qué punto estos significados están determinados por experiencias que se encuentran marcadas por la interacción/intersección de los factores de clase, género, raza, sexualidad y país de procedencia (en el caso de ser migrante).

Desde estas aportaciones, se considera la agencia como un elemento clave a la hora de pensar y teorizar sobre el devenir migratorio femenino, debido a que otorga nuevos elementos que sitúan la acción individual y colectiva como eje relevante en los momentos donde se va construyendo un nuevo "habitus" y/o entorno social, imprimiendo un sello identitario que determina la forma en que se percibe y actúa en la realidad social. En palabras de Bourdieu (2007) el mundo social se caracteriza por estructuras objetivas que orientan y/o coaccionan las prácticas o representaciones. De ahí que al momento de situar los procesos migratorios femeninos y la complejidad que ello injiere en la vida de éstas, la agencia se estructura, a nivel teórico-epistemológico, como un locus necesario de abordar, pues permite debilitar estereotipos y homogenizaciones que se erigen en torno a la figura de la mujer migrante y que no permite 
visibilizar las prácticas y capacidad de acción frente al acontecer que implica situarse, no sólo en un nuevo contexto social, sino también en un campo personal que envuelve nuevos sistemas simbólicos y patrones culturales a los cuales deben emplazar sus cuerpos, subjetividades e identidades sociales y culturales.

\section{MODELO DE ANÁLISIS: UNA APROXIMACIÓN A LA AGENCIA FEMENINA}

Los apartados anteriores desarrollan aquellas aportaciones teóricoconceptuales en torno a los procesos migratorios. A continuación se presentan aquellos elementos que buscan conectar cómo se despliega la agencia femenina en el proceso migratorio y de qué manera ello permite enfrentar dificultades propias del contexto, las preocupaciones familiares, los obstáculos para lograr los objetivos y propósitos, las estrategias de supervivencias, etc. Para aproximarnos a este objetivo, vamos a presentar un modelo de análisis que surge de las reflexiones teóricas anteriores y de los hallazgos preliminares del trabajo empírico; trabajo que se interroga sobre cómo, al tensionar los procesos de inclusión/exclusión, se genera una sinergia experiencial que permite pensar y situar la agencia y lo que deriva de ella. Pero antes es necesario puntualizar algunas cuestiones metodológicas.

\subsection{Apuntes metodológicos}

El trabajo empírico de esta investigación se llevó a cabo por medio de una aproximación cualitativa a veinticinco mujeres latinoamericanas en la ciudad de Barcelona entre los años 2015 y 2016. Dicha aproximación permitió un acercamiento a los discursos del colectivo de estudio, los significados y las construcciones sociales de sus experiencias migratorias. En cuanto a la técnica de recogida de información, se emplearon entrevistas semiestructuradas, comprendidas como una técnica que mejor se adecuaba a la hora de aproximarse a las experiencias vividas, tanto de su presente como de su pasado. Para el análisis de la información, se trabajó con el Análisis Crítico del Discurso Feminista (ACDF), ya que permite "avanzar en una comprensión más rica y matizada de los complejos mecanismos del poder e ideología en el discurso y en el sostenimiento (jerárquicamente) social del género" (Lazar 2007: 141).

Desde este enfoque, surgen los interrogantes sobre cómo las mujeres han sido historizadas, cómo se expresan en los discursos las representaciones sociales y qué lugar ocupan en la enunciación discursiva. Estas interrogantes son atingentes cuando el propósito es observar cómo las relaciones asimétricas de poder emergen de los discursos marcados por su connotación genérica. Para ello, es necesario no sólo situar el discurso en un determinado contexto, sino también observar/analizar como éste se expresa, señala, constituye y legitima la desigualdad social. 
En relación a la selección de las mujeres entrevistadas, se estableció un casillero tipológico orientado a controlar una mayor heterogeneidad de la muestra, y por tanto una mayor variabilidad en los discursos. Se definieron dos criterios de selección (buscando evitar posibles sesgos): edad y años de residencia. En cuanto a la edad, se precisaron los tres grupos etarios más representativos en edad laboral: 24 -34 años; 35-44 años y 45 años y más (Parella, Petroff, 2014). Los años de residencia se dividieron en dos: menos de tres años y más de tres años. Esto se fundamentó según lo estipulado el Reglamento de la Ley Orgánica 4/2000 sobre derechos y libertades de los extranjeros en España y su integración social, el cual señala que para poder acceder a residencia por arraigo social se debe acreditar la permanencia continua en el país por más de tres años. Si bien estos fueron los criterios para seleccionar la muestra, a la hora sistematizar la información afloraron factores que enriquecieron el análisis, permitiendo observar la heterogeneidad y complejidad de la muestra. Así por ejemplo, el nivel educativo, la inserción en el mercado laboral, la situación administrativa y los objetivos migratorios, por separado y en conjunto, reflejan una amplia gama de situaciones y escenarios que facilitan debilitar imaginarios homogéneos y estereotipados. A su vez, ello permite comprender el desarrollo de estrategias diversas y las forman de enfrentar las complejidades del proceso migratorio.

\subsection{Modelo de análisis: propuesta teórica-experiencial para aproximarse a la agencia femenina en contextos migratorios}

Para aproximarnos a la agencia se ha definido un modelo de análisis que se estructura a partir de tres ejes transversales: procesos de inclusión, exclusión y agencia, los cuales actúan como conector teórico-epistemológico frente a la conformación de nuevas realidades y/o subjetividades. Pues bien, desde los planteamientos teóricos ya trazados consideramos útil -para pensar el despliegue de la agencia femenina en contextos migratorios- el paradigma de la interseccionalidad, pues nos permite entender de qué manera se va conformando una matriz de desigualdad, violencia y opresión, ya sea por los condicionantes socio-históricos o por las dinámicas y tensiones propias de la estructura social del país de destino. Abordar la realidad de las mujeres migrantes implica pensar y situarse más allá de la categoría de género. Si bien es una categoría fundamental, es necesario incorporar otras que permitan introducir estos contextos. En este sentido, Crenshaw (1989) apunta que para entender cómo se estructura la racialización hacia los grupos oprimidos (en especial hacia las mujeres), es necesario explorar en las estructuras, procesos y representaciones sociales, para así aproximarse al impacto político y social que tiene la subordinación más allá de una lectura centrada en las implicancias del género. Esta lectura permite entender cómo las mujeres migradas se encuentran en una intersección donde su identidad racial, de clase y género va definiendo un mapa situacional donde convergen la historia, los contextos y circunstancias 
sociales y personales que puntualizan distintos niveles de dominación y jerarquización.

Ahora bien, como ya se ha mencionado, nuestra propuesta surge de las distintas contribuciones teóricas que han profundizado en los procesos migratorios femeninos, pero también de las voces de las mujeres que participaron en esta investigación; por consiguiente, es fruto tanto de la teoría como de las experiencias vividas que han emergido en el trabajo empírico. El objetivo es enlazar, en una primera instancia, los distintos espacios/momentos al proceso migratorio: los momentos previos a emigrar y la manera en que las motivaciones van marcando la hoja de ruta; la constitución de un espacio transnacional que emerge (a nuestro entender) una vez superado el duelo migratorio y que define una posición multi-situada que proyecta influencias en ambos lados de la frontera; posteriormente, tras el asentamiento, el desarrollo de estrategias de supervivencia económica y estrategias asociativas a nivel social. Asimismo, estos momentos son vividos o enfrentados de maneras diversas por las participantes, pues sus características sociales, económicas y/o educativas las llevan a conformar un dinamismo heterogéneo que se intersecta e interactúa de forma circular. Así por ejemplo, en el análisis destacan cuatro elementos que dan soporte a un análisis complejo y múltiple: nivel educativo, inserción en el mercado laboral, situación administrativa y objetivos migratorios (por motivos económicos, desarrollo personal o puerta de escape a la violencia-social, económica, sexual, machista- en sus países de origen). Tales dimensiones y/o factores inciden, definen o delimitan una ruta en su devenir migratorio.

Con respecto al análisis pre-migratorio observamos cómo las inquietudes y desafíos personales, las necesidades y requerimientos familiares e incluso las contradicciones y distensiones del contexto del país de procedencia, influyen en la decisión de emigrar. En este sentido, el contexto del país de origen puede ser entendido como un factor indirecto, en tanto que al comparar ambas realidades sociales (origen/destino), las participantes han podido visualizar cómo la normalización de la violencia estructural de sus países no les permitía comprender que eran justamente estas violencias las que incidían en sus condiciones de vida, en el acceso o limitación de oportunidades, en la precariedad, etc. Traer el pasado al presente es recurrente en los discursos, la comparación genera un subtexto de la realidad, una confluencia histórica que coloca la experiencia dentro de una continuidad que anhela la construcción de un futuro mejor o diferente. Dentro de esta lógica discursiva, si bien existe añoranza del pasado también hay una resistencia a las experiencias de violencia, desigualdad o todo aquello que representa desajuste social. Así lo relata una de las participantes al recordar su país (Uruguay):

... una ciudad desigual que es pequeña y aun así no se pueden distribuir bien los recursos (...), y a su vez esta inseguridad general que hay, porque una no tiene la libertad de caminar a cualquier hora por donde le apetezca y evitar ciertas calles por ciertas horas, y evitar caminar por el parque por la 
noche y eso lo sentía como una limitación bastante fuerte. (Serena ${ }^{3}, 34$ años, procedente de Uruguay y 9 años de residencia)

La distancia y el tiempo tensionan la realidad al confrontar aspectos vinculados a ambas localidades, lo cual es potenciado -en este caso- por las posibilidades personales y económicas que el nuevo contexto social le ofrece y que dista mucho a las cuales pudieron acceder en el país de procedencia. Con ello adquiere relevancia la multi-causalidad migratoria, pues conecta aspectos personales, sociales, creencias y representaciones que han interactuado como dispositivo migratorio. En ella circula una dualidad histórica que confronta el tiempo presente y la historia pasada, es decir, un presente que deviene pasado y que confiere una nueva temporalidad a las experiencias vividas.

En esta etapa las expectativas también actúan como un motor que direcciona las decisiones a corto y mediano plazo; expectativas que se apoyan y refuerzan por las redes migratorias. Éstas, para el caso de las entrevistadas, están conformadas por familias, amigos o conocidos que permiten establecer vínculos, conocimiento e información del país de destino, ayudando a amortiguar las condiciones de llegada y posterior asentamiento. Así lo manifiesta una entrevistada:

(...) aquí tenía a mi familia y a mis amigos y no me sentí sola. Aquí tenía a toda mi gente; en ese periodo no había soledad (...), nadie se podía sentir solo; te llevaban a los bares, te llevaban por allí, te enseñaban, te daban dinero para que te compres tu tarjeta. Normalmente era tu gente, tus tios, hermanos, etc.; siempre había mucho apoyo (María, 51 años, procedente de República Dominicana y 15 años de residencia).

En el espacio/momento de asentamiento, tal y como se observa en el modelo de análisis, comienzan a aparecer sentimientos de soledad y desarraigo fruto de la separación y pérdida de vínculos directos, generando altibajos que afrontan en su proceso de adaptación/integración y que se ha definido como duelo migratorio o "síndrome del inmigrante" (Achotegui, 2009). Así lo expresa una de las entrevistadas:

La primera etapa en mi caso fue muy buena pero pasado unos meses empecé como a sentir un duelo migratorio súper importante con muchísima melancolía (Estrella, 32 años, procedente de Argentina y 5 años de residencia).

A su vez estos sentimientos se agudizan por las fronteras simbólicas que diferencian, separan y también segregan a los "otros" con un "nosotros"; fronteras que se configuran alrededor de los "cuerpos migrados femeninos" tes.

${ }^{3}$ Los nombres utilizados son seudónimos para respetar la confidencialidad de las participan-

${ }^{4}$ Se opta por indicar procedencia en vez de nacionalidad, pues que algunas de las participantes han accedido a la nacionalidad española o tienen otra a parte de la de origen. 
y que impulsan una representación figurativa que viene de una raigambre histórica y cultural que alimenta estereotipos. Asimismo, en esta etapa es cuando la desterritorialización (simbólica y práctica) se hace efectiva a causa de la ausencia de vínculos sociales, culturales y de la propia identidad nacional, pero que como contra respuesta busca espacios de apropiación y pertenencia a una comunidad de afinidad que permite situar dinámicas de re-territorialización. En este sentido, el cuerpo -entendido como territorio des-localizado- alberga historias y memorias que se re-articula a través de interacciones múltiples que permiten el desarrollo de nuevos espacios relacionales que resignifican lo identitario nacional.

Por otra parte, el modelo de análisis nos evidencia las tensiones que generan los procesos de exclusión e inclusión. Es importante señalar que estar excluida no implica que no existan espacios de inclusión y viceversa; y ello porque cuando hablamos de procesos buscamos romper la visión dicotómica de la inclusión y exclusión.

En cuanto a los procesos de exclusión, el modelo presenta factores propios de la estructura social e institucional que se expresan en una dimensión económica a través de la discriminación y abuso laboral, la economía sumergida o informal, el desempleo o bajos salarios; en una dimensión política que se refleja en la limitación de derechos civiles (por ejemplo, el derecho al voto) o sociales (prestaciones); en una dimensión social por medio de la estigmatización, estereotipos sexistas y en la formación de nichos sociales endogámicos en determinados colectivos. En este sentido y relacionado con la exclusión social, emerge del análisis la relación entre el nivel educativo e inserción laboral. Dicha relación refleja que más allá del capital socioeducativo, el mercado laboral está altamente segmentado y "etnificado", por lo que las posibilidades de acceso a un trabajo relacionado con la formación profesional está limitado y orientado básicamente al servicio doméstico, cuidados y servicios. Son varios los ejemplos que pudimos recoger: profesoras, médicas, doctoras en ciencias, artistas, entre otras profesiones, que no podían ejercer en la ciudad de destino.

Otro ejemplo vinculado con la exclusión se relaciona con los derechos civiles. Una de las entrevistadas manifiesta que:

(...) Me cuesta mucho entender que para tener derecho a voto tienes que tener la nacionalidad, pero ¿tú sabes lo que cuesta eso? Han sido años de trabajo y aún no la tengo (Rocío, 35 años, procedente de Bolivia y 8 años de residencia).

En este relato, si bien la participante hace alusión a la limitación política que implica no tener la nacionalidad española, en otros ejemplos, es posible observar las restricciones sociales y laborales asociadas a la situación administrativa irregular, ya que al no tener los "papeles al día" se dificulta y condiciona el acceso a un mercado laboral regular, a prestaciones sociales, a viajar dentro o fuera de espacio Schengen, al miedo a ser interceptada por la policía y solicitar la documentación, el miedo a la deportación o expulsión del territorio, entre otras cuestiones. 
Esta situación lleva a las participantes, por un lado, a entender lo que implica ser inmigrante y las dificultades añadidas en caso de no tener regularizada la situación administrativa, y por otro, a desarrollar estrategias o posicionamientos para enfrentar tales limitaciones/dificultades.

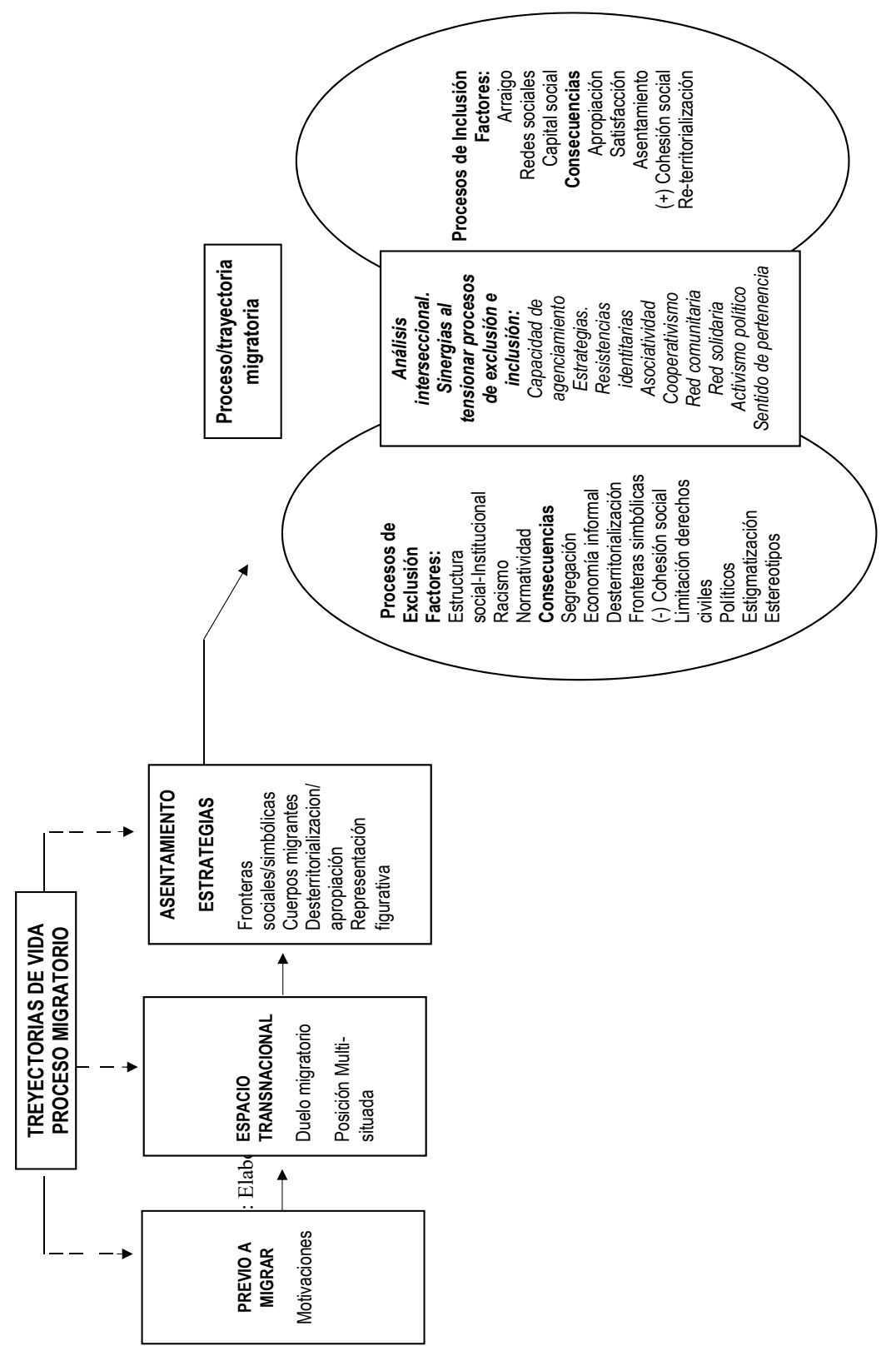


Por otra parte, los procesos de inclusión se expresan en dimensiones tales como el arraigo social, en las redes socioculturales, familiares y laborales que implican una apropiación espacial y pertenencia a una comunidad de afinidad identitaria (sea nacional o continental), en el capital social que promueve la inserción en distintos espacios y que extiende los niveles de cohesión social; en la re-territorialización. Esta última se refleja en el siguiente relato:

A pesar de lo difícil que ha sido vivir lejos de mi familia, siento que ahora tengo varias patrias, mi pertenencia es más amplia, me siento ecuatoriana, catalana, española y latinoamericana. Es como si todo fuera a la vez (María, 51 años, procedente de República Dominicana y 15 años de residencia).

En los procesos de re-territorialización se observa una confluencia de factores que permiten definir o trazar la significancia que adquieren las comunidades - espacios relacionales que otorgan identidad y pertenencia - más allá de aquellas establecidas por la nacionalidad de origen. Dichos factores se agrupan en: años de residencia, las experiencias adquiridas, la vinculación con comunidades y/o redes sociales, el nivel educativo o capital social, entre otros. A partir de estas evidencias nos preguntamos cómo, frente a las dificultades o determinadas facilidades sociales, las mujeres actúan y de qué manera proyectan sus aspiraciones o el logro de sus objetivos. Pues bien, a partir de la tensión de ambos procesos y considerando la perspectiva de la interseccionalidad, podemos advertir -en el modelo propuesto- un espacio donde se proyecta una capacidad de agenciamiento, una disposición de acción, que por las dinámicas propias del nuevo contexto que habitan, las incita a desarrollar estrategias de supervivencia personal y estrategias de resistencias identitarias. Estas últimas, como un desafío a no olvidar quiénes son y con la firme convicción de que la migración -para algunas- es un paso para alcanzar determinadas metas. La asociatividad y la expansión de redes sociales y laborales también son pensadas como estrategias para sobrevivir a la desterritorialización y falta de vínculos. Así, por ejemplo, una de las entrevistadas nos expresa lo siguiente:

He buscado espacios donde poder hacer cosas que me recuerdan y hacen sentir que no estoy lejos de mi tierra, de mi gente; no sé si te pasó a ti, pero en un momento necesitaba revivir y sentir mis raíces, y eso lo encontré con personas latinas, porque entre nosotros no existen diferencias (América, 34 años, procedente de Bolivia y 9 años de residencia).

Asimismo, otra forma de agenciamiento se vislumbra a través del cooperativismo presente en algunas asociaciones del tercer sector, las cuales permiten construir espacios económicos alternativos que facilitan disponer de una red comunitaria solidaria que también otorga sentido de pertenencia. De igual modo, cabe añadir que la mayoría de las participantes tienen enlazadas sus vidas con historias personales, familiares y sociales del país o lugar de procedencia, lo cual connota una conjunción transnacional a dichas actitudes y acciones que fortalecen su agencia. 


\section{CONCLUSIÓN}

La migración femenina desde hace décadas ha comenzado a ser un campo muy fructífero dentro de las ciencias sociales, desarrollando distintos marcos analíticos, enfoques epistemológicos y orientaciones metodológicas. Ello ha permitido alcanzar un significativo marco de referencia a la hora de investigar sobre este tema. Desde las distintas contribuciones, las investigaciones sobre género y migración han logrado visibilizar las construcciones homogéneas y universalistas que se edifican sobre las mujeres inmigrantes y que no permiten comprender, por ejemplo, el protagonismo que han alcanzado en tal proceso. Ello ha implicado que los enfoques androcéntricos, que las situaban como actor secundario, se debiliten debido a que en la actualidad las perspectivas teóricas profundizan en un análisis que incorpora las distintas etapas que las mujeres enfrentan en el proceso migratorio y las consecuencias que ello implica a nivel personal, familiar, social y laboral. En este sentido, las contribuciones de la perspectiva transnacional han permitido comprender la influencia de la multiposionalidad en la vida de las mujeres que emigran, la manera en que esta posición actúa a la hora de resignificar sus cuerpos e identidades y la forma en que la bidireccionalidad actúa como catalizar subjetivo y objetivo a la hora de repensarse y situarse en el "aquî́" y "allá".

Es entonces que al momento de analizar las experiencias migratorias de las mujeres, es importante considerar no sólo las motivaciones y/o proyectos que las incitaron a asumir tal decisión, sino también las distintas estrategias que desplegaron antes de emigrar como una vez instaladas en el país de destino; estrategias que invitan a resituar la agencia como eje dentro de los estudios en el área. Es en esta línea y recapitulando las distintas perspectivas teóricas, que valoramos la relevancia analítica que aporta la agencia, puesto que permite conocer y profundizar los momentos/etapas del proceso migratorio que transitan las mujeres; la forma en que despliegan mecanismos para alcanzar sus objetivos, las estrategias de supervivencia social, personal, familiar y laboral; la capacidad asociativa como dispositivo de resistencias identitarias, la manera en que asumen las dificultades propias del nuevo contexto social del país de destino; la (re) significación de los roles de género; la actitud frente a la desterritorialización, etc. Es decir, la agencia confiere una mirada hacia estos "otros" cuerpos que se reconstruyen y redefinen en este proceso y que requieren ser pensados más allá de categorías pre-establecidas, pues así se podrá observar la heterogeneidad de sus procesos vitales y la manera en estos se intersectan con sus experiencias migratorias; todo ello marcado por elementos que actúan en la conformación de múltiples sistemas de discriminación y violencias. En este sentido, el paradigma de la interseccionalidad se instala como un enfoque epistemológico que permite entender cómo, y a pesar de la confirmación de una matriz de opresión, se despliega una capacidad agencial; cómo, por ejemplo, frente al racismo se activan acciones de resistencias identitarias, que, si bien no entran en confrontación con la cultura del país de destino, persiguen el reconocimiento de un "otro" que comporta una cultura diferente y que puede convivir con la cultura 
local.

Por tanto, nuestra propuesta busca aportar en el área de los estudios de migración, una nueva lectura sobre los procesos migratorios femeninos; lectura que pretende pensar cómo, más allá de un enfoque genealógico, se pueden considerar las experiencias y trayectorias de vida como elementos significativos que inciden en sus itinerarios como migrantes. Desde nuestra perspectiva, las experiencias, motivaciones, proyectos y estrategias se amalgaman en un escenario desde donde se resalta la capacidad de acción, la actitud frente a la necesidad no sólo de alcanzar los objetivos, sino de subsistir, de asumir y enfrentar la realidad o el nuevo "habitus" social. Desde la capacidad de agenciamiento de las mujeres migrantes, es posible aproximarnos a las distintas problemáticas que enfrentan y cómo lo resuelven en lo cotidiano; nos permite dar un salto cualitativo a la hora generar taxonomías que -muchas veces- se alejan de sus propias historias y experiencias y que tiende a establecer axiomas que pueden escapar de la realidad o de sus realidades.

\section{BIBLIOGRAFÍA}

ACHOTEGUI, J. (2009): "Migración y salud mental. El síndrome del inmigrante con estrés crónico y múltiple (síndrome de Ulises)", Zerbitzuan, 46, pp. 163-171.

ACIÉN, E. (2009): "Sexualidad, prostitución y migrantes. Concepciones, posiciones y realidad", en Las migraciones en el mundo: desafíos y esperanzas, Barcelona, Icaria, pp. 251-272.

ALCAIDE, V. (2013): El capital social y las trayectorias laborales. Las redes personales como mecanismos y recursos para la inserción y la trayectoria laboral, disponible en http://www.tdx.cat/bitstream/handle/10803/129414/val1de1.pdf?sequence=1 [consulta: 15-6-2016]

ALKIRE, S. (2005): "Subjective quantitative studies of human agency", Social Indicators Research, vol. 74 (1), pp. 217-260.

ALKIRE, S. (2008): Concepts and measures of agency, University Oxford, Oxford Poverty \& Human Development Initiative.

ANZALDÚA, G. (1987): Borderlands/La Frontera: the New Mestiza, San Francisco, Spinsters/Aunt Lute.

AUBARELL, G. (2000): "Una propuesta de recorrido bibliográfico por las migraciones femeninas en España”, Papers: Revista de Sociología, 60, pp. 391-413.

BOBES, V. (2012): "El transnacionalismo como enfoque. Una reflexión para construir un modelo analítico", en Debates sobre el transnacionalismo, México, Flacso, pp. 6-20.

BOURDIEU, P. (1985): "The forms of capital", en Handbook of theory and research for the sociology of education, Nueva York, Greenwood, pp. 241-258.

BOURDIEU, P- (2007): Cosas dichas, Barcelona, Gedisa.

BRANDARIZ, J. (2009). "La funcionalidad de la construcción de los migrantes como sujetos de riesgo en el sistema penal español. Derecho Penal del Enemigo, gestión de la exclusión e inclusión subordinada", en Constitución, derechos fundamentales y sistema penal, Valencia, Tirant lo Balch, pp. 287-306. 
BRIGGS, L.; MCCORMICK, G.; WAY, J. (2008): “Transnationalism: A category of analysis", American Quartely, vol. 60 (3), pp. 635-648.

CALAVITA, K. (2006): "Gender, migration and law: crossing borders and bridging disciplines", International Migration Review, vol. 40 (1), pp. 104-132.

CARRASCO, C. (2013): "El cuidado como eje vertebrador de una nueva economía", Cuadernos de relaciones Laborales, vol. 31 (1), pp. 39-56.

CARRERA, S. (2006): "Programas de integración para inmigrantes: Una perspectiva comparada en la Unión Europea", Migraciones, 20, pp. 37-73.

CASADO, E. (2003): "La emergencia del género y su resignificación en tiempos de los "post", Foro Interno, 3, pp. 41-65.

CASADO, E. (1999): “A vueltas con el sujeto del feminismo”, Política y sociedad, 30, pp. 73-92

CASTLES, S.; MILLER, M. (1998): The age of migration international population movements on the modern world, Nueva York, The Guilford Press.

CATARINO, C., OSO, L. (2000): "La inmigración femenina en Madrid y Lisboa", Papers: Revista de Sociología, 60, pp.183-207.

COLEMAN, J. (1990): Foundations of Social Theory, Harvard University Press.

CRENSAW, K. (1989): "Demarginalizing the intersection of Race and Sex: A black feminist critique of antidiscrimination doctrine, feminist theory and antiracist politics", University of Chicago Legal Forum, 1, pp. 139-167.

DE GIORGI, A. (2010): "Immigration control, post-Fordism, and less eligibility. A materialist critique of the criminalization of immigration across Europe", Punishment \& Society, vol. 12 (2), pp. 147-167.

DUMITRU, S. (2014): "From "brain drain" to "care drain": Women's labor migration and methodological sexism", Women's Studies international Forum, 47, pp. 203212.

ESCRIVÁ, A. (2000): “Empleadas de por vida?”, Papers: Revista de Sociología, 60, pp. 327-342.

FLEURY, S. (1998): "La política social, exclusión y equidad en América Latina en los años noventa", Nueva Sociedad, 156, pp. 1-25.

FOESSA (2014): VII Informe sobre exclusión y desarrollo social en España, Madrid, Foessa.

GIL ARAUJO, S. (2005): "Cartografías migratorias: migraciones internacionales en el marco de las relaciones Norte-Sur", en La migración un camino entre el desarrollo y la cooperación, Madrid, Cip-Fuhem, pp.13-52

GODOY, L. (2007): "Fenómenos migratorios y género: identidades femeninas remodeladas", PSYKHE, 16, pp. 41-51.

GONZÁLEZ y GÜEL (2012): “Sentidos subjetivos: su rol estructural en las políticas de inclusión”, Revista del CLAD, Reforma y Democracia, 53, pp. 1-8

GREGORIO GIL, C. (1998): Migración Femenina: su impacto en las relaciones de género, Narcea Ediciones.

GREGORIO GIL, C. (1999): "Los movimientos migratorios del sur al norte como procesos de género", en Globalización y Género, Madrid, Editorial Síntesis, pp. 259-288.

GREGORIO GIL, C. (2009): "Mujeres inmigrantes: colonizando sus cuerpos mediante fronteras procreativas, étnico-culturales, sexuales y reproductivas", Por una izquierda alternativa, vol. 18 (104), pp.42-54.

GUARNIZO, L. (2004), “Aspectos económicos del vivir transnacional”, Colombia Internacional, 59, pp.12-47. 
HERRERA, G. (2012): "Repensar el cuidado a través de la migración internacional: mercado laboral, Estado y familias transnacionales en Ecuador", Cuadernos de Relaciones Laborales, vol. 30 (1), pp.139-159.

HERRERA, G. (2013): "La migración vista desde el lugar de origen", Íconos-Revista de Ciencias sociales, 15 , pp. 86-94.

HONDAGNEU-SOTELO, P. (2007): “La incorporación del género a la migración: no sólo para feministas no sólo para la familia", en El país transnacional: migración mexicana y cambio social a través de la frontera, México D.F., Universidad Autónoma de México, pp. 423-451.

HONDAGNEU-SOTELO, P. (2011): "Gender and migration scholarship: an overview from a $21^{\text {st }}$. century perspective”, Migraciones Internacionales, vol. 6 (1), pp. 219233.

HONDAGNEU-SOTELO, P.; AVILA, E. (1997): "I'm here, but I'm there" The meanings of Latina transnational motherhood", Gender \& Society, vol. 11 (5), pp. 548-571.

JIMÉNEZ, C. (2010): “Transnacionalismo y migraciones: aportaciones desde la teoría de Pierre Bourdieu", Empiria, Revista de metodología y Ciencias Sociales, 20, pp. 13-38

LAPARRA, M., et al. (2007): “Una propuesta de consenso sobre el concepto de exclusión. Implicaciones metodológicas", Revista española de tercer sector, 5, pp. $15-57$.

LAZAR, M. (2007): "Feminist critical discourse analysis: Articulating a feminist discourse praxis", Critical Discourse Studies, vol. 4 (2), pp.141-164.

LECINANA, M. (2004): "La crisis del sujeto desde el feminismo filosófico". V Jornadas de Investigación en Filosofía, disponible en https://www.aacademica.org/000094/72.pdf [consulta: 05-05-2015]

LEVITT, P.; GLICK SCHILLER, N. (2004): "Conceptual and methodological development in the study of international migration", International Migration Review, vol. 38 (3), pp. 1002-1039.

MAGLIANO, M.; ROMANO, S. (2009): "Migración, género y (sub) desarrollo en la agenda política internacional: Una aproximación crítica desde la periferia sudamericana", Cuadernos del Cendes, 72, pp. 101-129.

MAHMOOD, S. (2008): "Teoría Feminista y el agente dócil: algunas reflexiones sobre el renacimiento islámico en Egipto", en Descolonizando el feminismo: Teorías y prácticas desde los márgenes, Madrid, Cátedra, pp.162-214.

MARTÍN, E. (2012): "Estrategias migratorias de las mujeres ecuatorianas en Sevilla: acumulación de capital social en tiempos de crisis", Migraciones Internacionales, 23, pp.107-139.

MEDINA MARTÍN, R. (2013): "Feminismos periféricos, feminismos-otros: una genealogía feminista decolonial por reivindicar", Revista internacional de pensamiento político, 8, pp. 53-79.

MICOLTA, A. (2005): "Teorías y conceptos asociados al estudio de las migraciones internacionales", Trabajo Social, 7, pp. 59-76.

MONTAÑEZ, P.; MOYANO, M. (2006): "Violencia de género sobre inmigración en España. Un análisis psicosocial", Pensamiento psicológico, vol. 2 (6), pp. 21.32.

OSO, L. (2003): Estrategias migratorias de las mujeres ecuatorianas y colombianas en situación irregular: servicio doméstico y prostitución en Galicia, Madrid y Pamplona, Coruña, Universidade da Coruña, Facultade de Socioloxia. 
PARELLA, S.; CAVALCANTI, L. (2010): "Dinámicas familiares transnacionales y migración femenina en el contexto migratorio boliviano", en Familias, niños, niñas y jóvenes migrantes. Rompiendo estereotipos, Madrid: IEPALA, pp. 93-106.

PARELLA, S.; PETROFF, A. (2014): "Migración de retorno en España: Salidas de inmigrantes y programas de retorno en un contexto de crisis", CIDOB Anuario de la inmigración en España, pp. 63-87.

PORTES, A. (1999): “Capital social: sus orígenes y aplicaciones en la sociología moderna", en De igual a igual. El desafío del Estado ante los nuevos problemas sociales, México: Fondo de Cultura Económica, pp. 243-266.

RUIZ, M. (1998): “Subjetividad femenina”, Espiral, 5(13), pp. 143-160.

SEGURA, D., ZAVELLA, P. (2007): Women and migration in the U.S- Mexico borderlands: a reader, Londres, Duke University Press.

SILVER, H. (1995): "Reconceptualizing social disadvantge: three paradigms of social exclusion", en Social exclusion: Rhetoric, reality, responses, Genova, International Institute for Labour Studies, pp, 57-80.

SINATTI, G. (2008): "Migraciones, transnacionalismo y locus de investigación: multi-localidad y la transición de "sitios" a "campos", en Nuevos retos del transnacionalismo en el estudio de las migraciones, Madrid, Subsecretaria General de Información Administrativa y Publicaciones del Ministerios de Trabajo e Inmigración, pp. 93-112.

SPIVAK, G. (1998): "Puede hablar el sujeto subaltern”, Orbis Tertius, vol. 3 (6), pp. $1-44$.

SUBIRATS, J., GOMÀ, R., BRUGUÉ, J. (2005): Análisis de los factores de exclusión social, Barcelona, Fundacióm BBVA, Institut d’Estudis Autonómics.

SUBIRATS J. et al. (2010): Ciudadanía e inclusión social. El tercer sector y las políticas públicas de acción social, Barcelona, Fundación Catalana L`Esplai

TAPIA, M. (2011): "Género y Migración: trayectorias investigativas en Iberoamérica”, Revista Encrucijada Americana, 2, pp. 115-147.

TRUJILLO BARBADILLO, G. (2009): "Del sujeto político la Mujer a la agencia de las (otras) mujeres: el impacto de la crítica queer en el feminismo del Estado Español”, Política y Sociedad, vol. 46 (1/2), pp. 161-172. 
